\title{
Use of whole genome sequencing of commensal Escherichia coli in pigs for antimicrobial resistance surveillance, United Kingdom, 2018
}

Emma Stubberfield ${ }^{1}$, Manal AbuOun¹, Ellie Sayers ${ }^{1,2}$, Heather M 0'Connor ${ }^{3}$, Roderick M Card ${ }^{1}$, Muna F Anjum ${ }^{1}$

1. Department of Bacteriology, Animal and Plant Health Agency, Weybridge, Surrey, United Kingdom

2. University of East Anglia/Quadram Institute Bioscience, Norwich Research Park, Norwich, United Kingdom

3. Department of Epidemiological Sciences, Animal and Plant Health Agency, Weybridge, Surrey, United Kingdom

Correspondence: Muna F Anjum (muna.anjum@apha.gov.uk)

Citation style for this article:

Stubberfield Emma, AbuOun Manal, Sayers Ellie, O'Connor Heather M, Card Roderick M, Anjum Muna F. Use of whole genome sequencing of commensal Escherichia coli in pigs for antimicrobial resistance surveillance, United Kingdom, 2018. Euro Surveill. 2019;24(50):pii=1900136. https://doi.0rg/10.2807/1560-7917.

ES.2019.24.50.1900136

Article submitted on 19 Feb 2019 / accepted on 01 Aug 2019 / published on 12 Dec 2019

Background: Surveillance of commensal Escherichia coli, a possible reservoir of antimicrobial resistance (AMR) genes, is important as they pose a risk to human and animal health. Most surveillance activities rely on phenotypic characterisation, but whole genome sequencing (WGS) presents an alternative. Aim: In this retrospective study, we tested $515 E$. coli isolated from pigs to evaluate the use of WGS to predict resistance phenotype. Methods: Minimum inhibitory concentration (MIC) was determined for nine antimicrobials of clinical and veterinary importance. Deviation from wild-type, fully-susceptible MIC was assessed using European Committee on Antimicrobial Susceptibility Testing (EUCAST) epidemiological cut-off (ECOFF) values. Presence of $A M R$ genes and mutations were determined using APHA SeqFinder. Statistical twoby-two table analysis and Cohen's kappa (k) test were applied to assess genotype and phenotype concordance. Results: Overall, correlation of WGS with susceptibility to the nine antimicrobials was $98.9 \%$ for test specificity, and $97.5 \%$ for the positive predictive value of a test. The overall kappa score $(k=0.914)$ indicated AMR gene presence was highly predictive of reduced susceptibility and showed excellent correlation with MIC. However, there was variation for each antimicrobial; five showed excellent correlation; four very good and one moderate. Suggested ECOFF adjustments increased concordance between genotypic data and kappa values for four antimicrobials. Conclusion: WGS is a powerful tool for accurately predicting AMR that can be used for national surveillance purposes. Additionally, it can detect resistance genes from a wider panel of antimicrobials whose phenotypes are currently not monitored but may be of importance in the future.

\section{Introduction}

Escherichia coli in animals comprise a diverse range of strains. They are a reservoir and indicator of antimicrobial resistance (AMR) genes that can be mobilised to other bacteria including zoonotic pathogens, and therefore pose a risk to human and animal health. Mobile elements such as plasmids and transposons are typically responsible for horizontal transfer of AMR genes [1,2]. Antimicrobial resistance can also be attributed to the expression of efflux pumps or single nucleotide polymorphisms (SNPS) present within chromosomal genes such as the DNA gyrase, that counteract the actions of certain antimicrobials [3].

Antimicrobial resistance is traditionally measured using phenotypic methods, for example, the minimal inhibitory concentration (MIC), and interpreted using breakpoints to determine resistance or susceptibility. The European Committee on Antimicrobial Susceptibility Testing (EUCAST) sets a clinical breakpoint that determines the likelihood of therapeutic success for treating infection; isolates with an MIC above this level are associated with a high level of therapeutic failure. EUCAST also defines an epidemiological cut-off (ECOFF) value which is used to differentiate the susceptible wild type bacterial population from non-wild type isolates with an acquired resistance mechanism. The European Union (EU) directive $2003 / 99 / E C$ requests the harmonised monitoring and reporting of $A M R$, which is coordinated by the European Food Safety Authority (EFSA) and European Centre for Disease Prevention and Control (ECDC), to be carried out by EU countries for joint reporting of animal, food and human data [4]. The EU countries perform antimicrobial susceptibility testing on selected pathogenic and indicator organisms, which include $E$. coli $[4,5]$, but no details are obtained on the underlying molecular mechanisms. 


\section{TABLE 1}

Antimicrobial resistance genes identified in Escherichia coli isolates from pooled caecal contents of healthy pigs, for nine antimicrobials, United Kingdom, $2018(\mathrm{n}=515)$

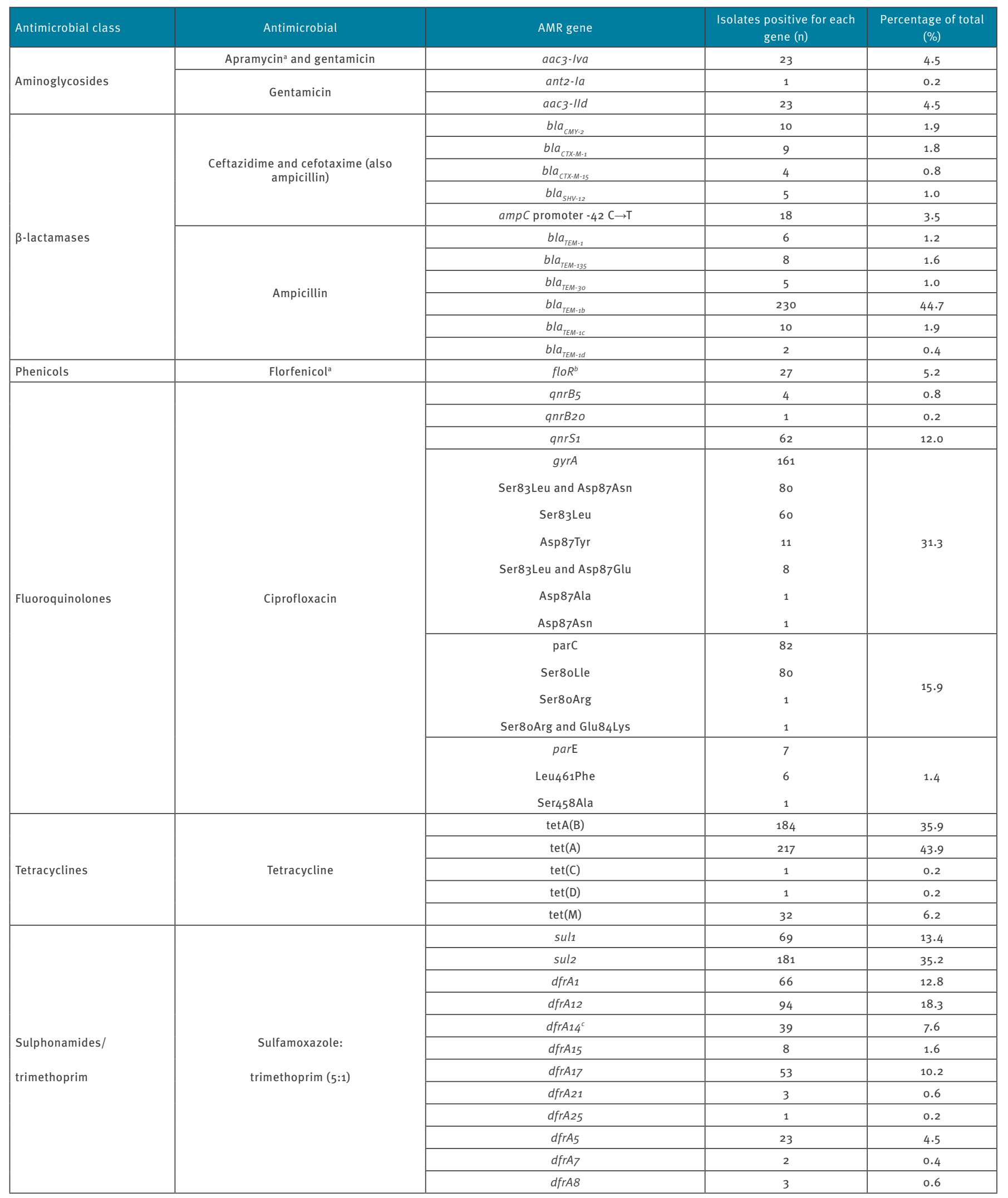

AMR: antimicrobial resistance.

${ }^{a}$ Apramycin and florfenicol are only used in veterinary medicine.

${ }^{b}$ floR gene presence was determined at $99 \%$ mapping.

${ }^{c} d f r A_{14}$, tet( $\left.A\right)$ and tet $(M)$ gene presence was determined at greater than $86 \%$ mapping. 
Correlation of whole genome sequencing and ECOFF or DANMAP values, test performances and kappa correlations for Escherichia coli isolates from pooled caecal contents of healthy pigs by antimicrobial, United Kingdom, 2018 ( $\mathrm{n}=515$ )

\begin{tabular}{|c|c|c|c|c|c|c|c|c|c|c|}
\hline \multirow{2}{*}{$\begin{array}{l}\text { Antibiotic } \\
\text { Cut-off } \\
(\mathrm{mg} / \mathrm{L})\end{array}$} & \multirow{2}{*}{$\begin{array}{l}\text { Ciprofloxacin } \\
\text { ECOFF (>0.06) }\end{array}$} & \multirow{2}{*}{$\begin{array}{l}\text { Cefotaxime } \\
\text { ECOFF } \\
(>0.25) \\
\end{array}$} & \multirow{2}{*}{$\begin{array}{l}\text { Ceftazidime } \\
\text { ECOFF (>0.5) }\end{array}$} & \multirow{2}{*}{$\begin{array}{l}\text { Gentamycin } \\
\text { ECOFF }(>2)\end{array}$} & \multirow{2}{*}{$\begin{array}{l}\text { Florfenicola }^{a} \\
\text { ECOFF }(>16)\end{array}$} & \multirow{2}{*}{$\begin{array}{l}\text { Ampicillin } \\
\text { ECOFF } \\
(>8) \\
\end{array}$} & \multirow{2}{*}{$\begin{array}{l}\text { Apramycin }^{\text {a }} \\
\text { DANMAP } \\
(>16)\end{array}$} & \multirow{2}{*}{$\begin{array}{l}\text { SXT } \\
\text { ECOFF } \\
(>1)\end{array}$} & \multirow{2}{*}{$\begin{array}{l}\text { Tetracycline } \\
\text { ECOFF (> 8) }\end{array}$} & \multirow[b]{2}{*}{ Overall } \\
\hline & & & & & & & & & & \\
\hline $\mathrm{P}+/ \mathrm{G}+$ & 218 & 45 & 45 & 45 & 27 & 283 & 23 & 258 & 387 & 1,330 \\
\hline P-/G- & 249 & 464 & 441 & 468 & 479 & 218 & 492 & 209 & 114 & 3,135 \\
\hline $\mathrm{G}+/ \mathrm{P}-$ & 10 & 1 & 1 & 0 & 0 & 2 & 0 & 15 & 6 & 34 \\
\hline $\mathrm{G}-/ \mathrm{P}+$ & 38 & 5 & 28 & 2 & 9 & 12 & 0 & 33 & 8 & 136 \\
\hline \multicolumn{11}{|c|}{ Test performances } \\
\hline Specificity & $96.1 \%$ & $99.8 \%$ & $99.8 \%$ & $100.0 \%$ & $100.0 \%$ & $99.1 \%$ & $100.0 \%$ & $93.3 \%$ & $95.0 \%$ & $98.9 \%$ \\
\hline Sensitivity & $85.2 \%$ & $90.0 \%$ & $61.6 \%$ & $95.7 \%$ & $75.0 \%$ & $95.9 \%$ & $100.0 \%$ & $88.7 \%$ & $98.0 \%$ & $90.7 \%$ \\
\hline PPV & $95.6 \%$ & $97.8 \%$ & $97.8 \%$ & $100.0 \%$ & $100.0 \%$ & $99.3 \%$ & $100.0 \%$ & $94.5 \%$ & $98.5 \%$ & $97.5 \%$ \\
\hline NPV & $86.8 \%$ & $98.9 \%$ & $94.0 \%$ & $99.6 \%$ & $98.2 \%$ & $94.8 \%$ & $100.0 \%$ & $86.4 \%$ & $93.4 \%$ & $95.8 \%$ \\
\hline \multicolumn{11}{|c|}{ Kappa correlations } \\
\hline Карра & 0.814 & 0.9431 & 0.726 & 0.976 & 0.848 & 0.945 & 1.000 & 0.812 & 0.930 & \\
\hline
\end{tabular}

DANMAP: Danish Integrated Antimicrobial Resistance Monitoring and Research Programme; ECOFF: European Committee on Antimicrobial Susceptibility Testing epidemiological cut-off; G+: gene/SNP present; G-: gene/SNP absent; NPV: negative predictive value; P+: phenotype resistant; P-: phenotype sensitive; PPV: positive predictive value; SXT: sulfamethoxazole:trimethoprim.

a Apramycin and florfenicol are only used in veterinary medicine.

Kappa correlation: almost perfect (>0.900), strong agreement (0.800-0.900) and moderate agreement (0.600-0.790).

The presence of AMR genes harboured by bacteria can be determined by a variety of molecular methods, including PCR, DNA microarray and whole genome sequencing (WGS) [1]. Unlike PCR and microarrays, WGS offers the advantage of being able to screen the bacterial genome for multiple genes and mutations associated with $A M R$, which can be used to predict phenotypic susceptibility to antimicrobials $[1,6]$ and to retrospectively detect newly identified AMR genes [7]. To identify AMR determinants, WGS data have to be screened against a database of AMR genes such as CARD or ResFinder $[8,9]$.

In a 2017 report by EUCAST, it was stated that there is poor or non-existent evidence for using WGS as a method to infer antimicrobial susceptibility accurately [6]. Several recent studies have attempted to predict antimicrobial susceptibility from WGS data for a variety of bacteria, including $E$. coli [10-14]. When compared with phenotypic data, the predictions showed high concordance between genotype and phenotype with overall specificity and sensitivity>95\% [3]. However, many of these studies on $E$. coli are from clinical or known multidrug-resistant (MDR) isolates, and are limited to small panels of isolates $(\leq 155)$.

We present an analysis on the association of gene presence from WGS with AMR phenotype in a large panel of commensal $E$. coli to predict their resistance to antimicrobials of human clinical and veterinary importance. We aimed to provide further evidence to support the use of WGS to enhance AMR surveillance and accurately predict antimicrobial susceptibility.

\section{Methods}

\section{Bacterial isolates and antimicrobial susceptibility testing}

In this retrospective study we compare phenotypic MIC values, interpreted using ECOFFs, with AMR genotypes in WGS data from $515 \mathrm{E}$. coli isolated from pooled caecal contents of healthy pigs collected at abattoir from 57 farms across the United Kingdom (UK) from 2014 to 2015 [15].

Isolates were selected on Brilliance UTI Agar (Oxoid, Basingstoke, UK) plates containing either $1 \mathrm{mg} / \mathrm{L}$ cefotaxime (CTX), $1 \mathrm{mg} / \mathrm{L}$ ciprofloxacin (CIP), no antibiotic (NoAB) and on Brilliance carbapenem-resistant Enterobacteriaceae (CRE) Agar (Oxoid). The British Society of Antimicrobial Chemotherapy (BSAC) agar dilution method [16] was used to test the susceptibility of each isolate against a panel of nine antimicrobials, of which two (apramycin and florfenicol) are only used in veterinary medicine. These antimicrobials span seven AMR classes of veterinary and/or human clinical relevance [17]: ampicillin (0.25-128 $\mathrm{mg} / \mathrm{L})$, apramycin (1-128 mg/L), cefotaxime (0.004-128 mg/L), ceftazidime (0.004-128 mg/L), ciprofloxacin (0.004-128 $\mathrm{mg} / \mathrm{L}$ ), florfenicol (0.25-128 mg/L), gentamicin (0.03$128 \mathrm{mg} / \mathrm{L}$ ), sulfamethoxazole:trimethoprim 5:1 (0.15$640 \mathrm{mg} / \mathrm{L}$ for sulfamethoxazole and $0.03-128 \mathrm{mg} / \mathrm{L}$ for trimethoprim) and tetracycline $(0.25-128 \mathrm{mg} / \mathrm{L})$. The MIC was defined as the lowest concentration that inhibited growth. Susceptibilities were interpreted using the EUCAST ECOFF values [18] because of a decision made by BSAC to migrate from BSAC to EUCAST methods was made after this study commenced [19]. 


\section{FIGURE 1}

Almost perfect correlation between phenotype and genotype of Escherichia coli isolates from pooled caecal contents of healthy pigs by antimicrobial, United Kingdom, $2018(\mathrm{n}=515)$

\section{A. Ampicillin}
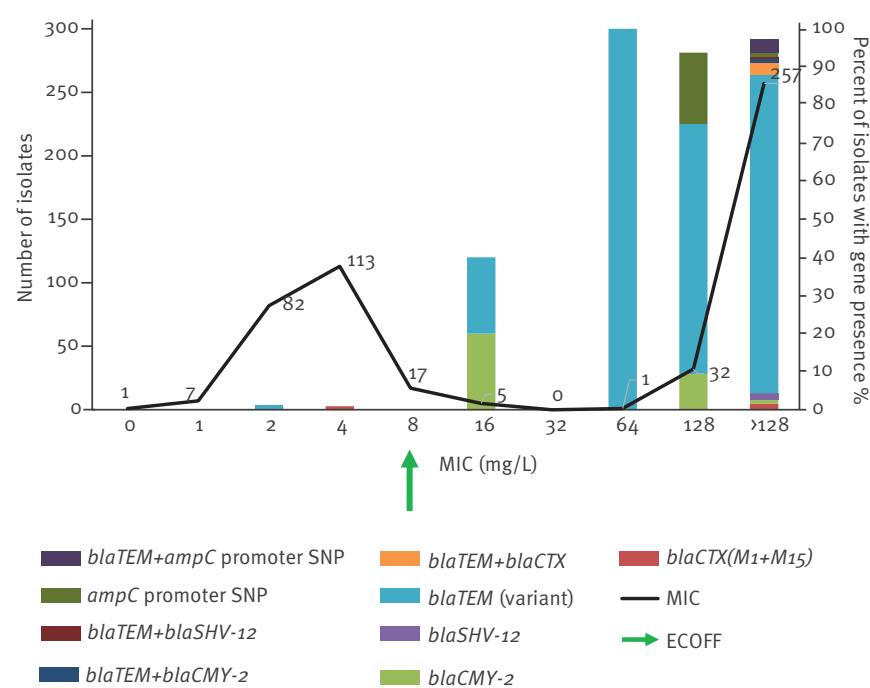

B. Cefotaxime
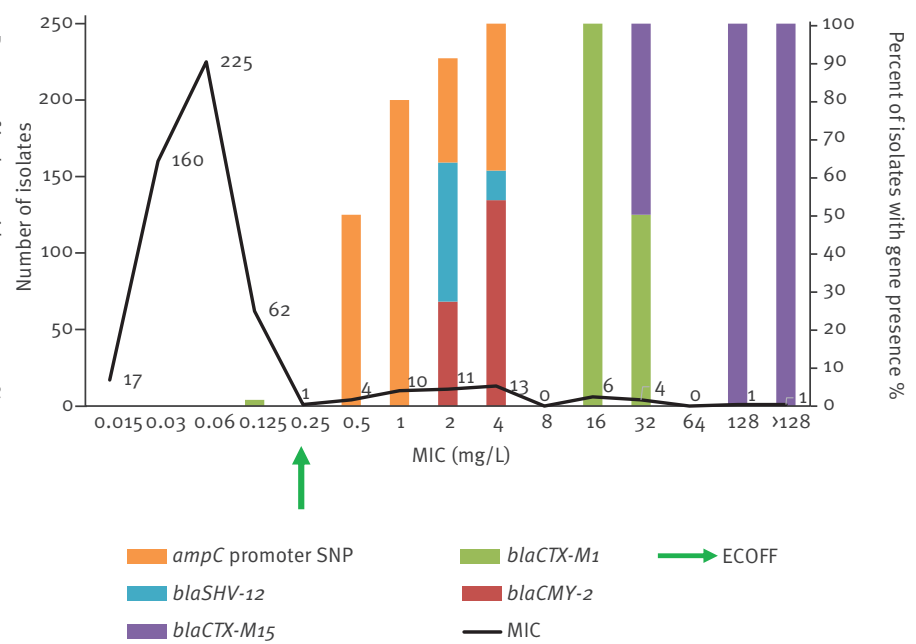

C. Apramycin

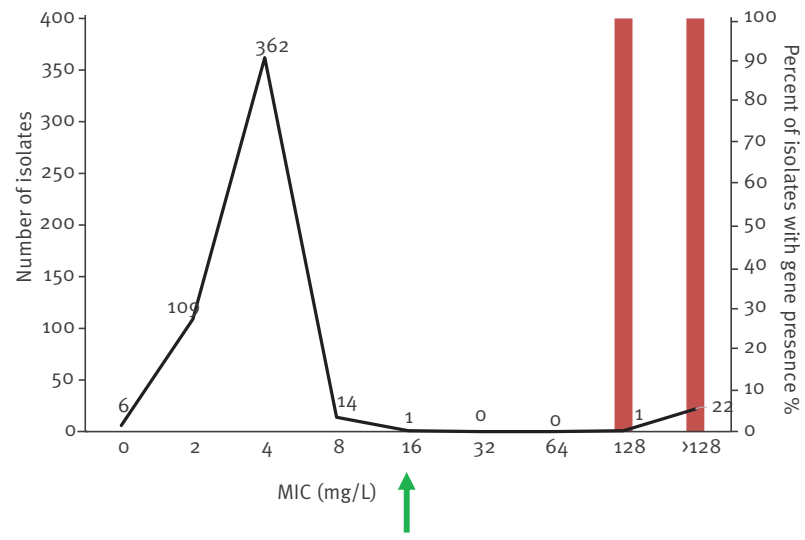

aac3-IVa $\longrightarrow$ DANMAP cut-off

- MIC
D. Gentamicin

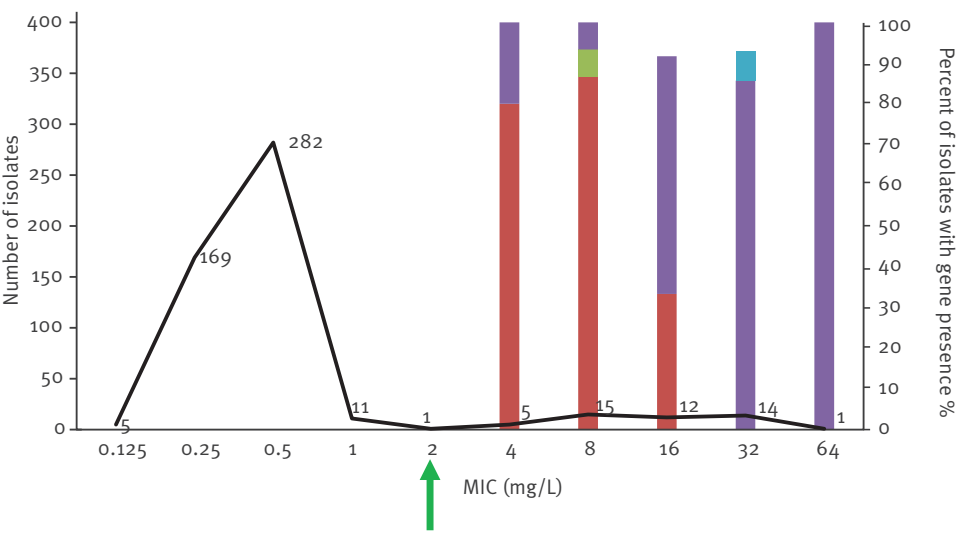

$\underset{a a c 3-I I d}{a \text { ant2-Ia }} \longrightarrow$ MIC

\section{E.Tetracycline}

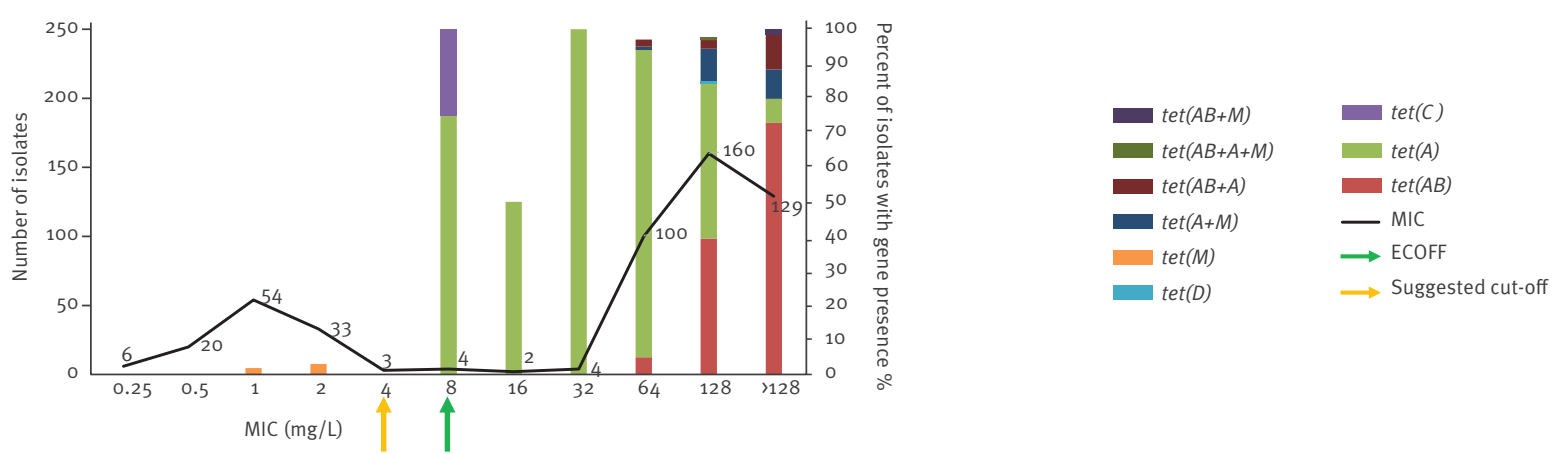

ECOFF: European Committee on Antimicrobial Susceptibility Testing epidemiological cut-off; MIC: minimum inhibitory concentration.

The MIC distribution and the underlying genetic mechanisms present in isolates are shown.

Apramycin (C) is only used in veterinary medicine. 
For apramycin, there is no defined ECOFF value or clinical breakpoint for $E$. coli so the Danish Integrated Antimicrobial Resistance Monitoring and Research Programme (DANMAP)-proposed breakpoint of $>16$ $\mathrm{mg} / \mathrm{L}$ was used [20]. The definition of reduced susceptibility refers to isolates with an MIC above the ECOFF value and susceptible isolates refers to isolates with an MIC equal to or less than the ECOFF value.

\section{Whole genome sequencing}

DNA was extracted and Illumina HiSeq 4000 System whole genome sequencing (WGS) (Illumina, San Diego, United States (US)) performed on the E. coli isolates and sequences were deposited in the European Nucleotide Archive (ENA) under study accession number PRJEB26317. The APHA SeqFinder pipeline was used to determine the presence of 2,044 AMR genes, including genes associated with the nine antimicrobials tested in this study [7]. Presence of AMR genes belonging to each AMR class considered was determined by mapping unassembled reads to a database of gene sequences, following quality control measurements described previously [7]. An AMR gene was considered present if there was $100 \%$ gene mapping to the reference in the APHA SeqFinder database of AMR genes, allowing up to 10 non-synonymous SNPS, with the exception of floR that was present at $99 \%$ gene mapping and $d f r A 14$, tet(A) a nd $\operatorname{tet}(M)$ that were present at greater than $86 \%$ gene mapping. Genome assembles were generated using SPAdes version 3.7.0 [21] and AMR gene presence was corroborated using abricate (https://github.com/tseemann/abricate) [21]. To identify SNPs in chromosomal genes/regions, gyrA, parC, parE and $\operatorname{ampC}$ promoter, associated with resistance (cSNP-AMR), ClustalW gene alignments were performed in DNASTAR Lasergene 11 Core Suite (DNASTAR Inc, Madison, US) and these were then incorporated into the APHA SeqFinder pipeline. $E$. coli strain K12 MG1655 was used as the reference.

\section{Statistical analysis}

The correlation between the presence/absence of AMR genes and/or associated SNPs from the WGS results and the susceptibility by MIC phenotypes was evaluated statistically by two-by-two table analysis, as performed previously for other phenotype/genotype correlations $[22,23]$ where test specificity, sensitivity and the positive predictive value (PPV) and negative predictive value (NPV) of the test were calculated using the following criteria: (i) correlation between WGSgene presence and MIC-resistant results as true positive (TP), (ii) WGS-negative and MIC-susceptible results as true negative (TN), (iii) WGS-gene presence but MICsusceptible results as false positive (FP) and (iv) WGSnegative but MIC-resistant results as false negative (FN). The capability of the MIC and WGS for identifying AMR was compared using the Cohen's kappa test (k), which is a measure of agreement above that expected by chance, with a $\mathrm{k}$ of $\mathrm{o}$ indicating that the test agrees as well as would be expected by chance, and a $\mathrm{k}$ of 1 indicating complete agreement. When assessing the kappa test, a result of above 0.900 was interpreted as almost perfect, $0.800-0.900$ as strong agreement, 0.600-0.790 as moderate agreement, $0.400-0.590$ as weak agreement and $0.200-0.390$ as minimal agreement [24].

\section{Results}

Minimum inhibitory concentration phenotype Of 515 E. coli tested, only 56 isolates (11\%) were susceptible to all antimicrobials tested, with the remaining isolates resistant to between one and seven antimicrobials. The most common reduced susceptibility was observed for tetracycline ( $77 \%, n=395)$, ampicillin (57\%, $\mathrm{n}=295)$ and sulfamethoxazole:trimethoprim $(57 \%, n=291)$. The least common reduced susceptibility was detected for the two veterinary antimicrobials included, florfenicol $(7 \%, n=36)$ and apramycin $(4.5 \%$, $n=23)$.

\section{Antimicrobial resistance genotype}

Seventy-eight of 515 isolates (15\%) had no detectable AMR genes or cSNP-AMR present in our database, with the remaining isolates having between one and nine acquired AMR genes and/or cSNP-AMR associated with the antimicrobials tested. Approximately $55 \%(n=283)$ of isolates harboured between three and seven AMR genes and/or cSNP-AMR showing a multidrug resistance genotype, with seven isolates harbouring genotypic resistances to all seven antimicrobial classes tested. The most common AMR genes included bla $a_{T E M}$. ${ }_{1 b}(45 \%), \operatorname{tet}(\mathrm{A})(44 \%)$ and tetA(B) (36\%) (Table 1), which corresponded with the high levels of reduced susceptibility to ampicillin and tetracycline detected by MIC.

\section{Genotypic prediction of resistance phenotype}

The relationship between genotype and phenotype was evaluated for the nine antimicrobials using the gene categories given in Table 1 . Using the ECOFF value, overall correlation of WGS with MIC was $99 \%$ for test specificity and $98 \%$ for the tests' PPV (Table 2). The kappa values for each antimicrobial tested ranged from 0.726 to 1.000 , and predominantly showed strong agreement $(K>0.800)$ between gene presence and reduced susceptibility (Table 2 ). The overall kappa score $(\mathrm{K}=0.914)$ indicated that WGS gene presence was highly predictive of reduced susceptibility and showed 'almost perfect' agreement with phenotypic MIC data from isolates. Test sensitivity was $91 \%$, which although lower than test specificity, still showed strong agreement and could be because of the presence of yet unknown genes/mechanisms that were absent from our database.

Almost perfect agreement, kappa value $>\mathbf{0 . 9 0 0}$ Five antimicrobials (ampicillin, cefotaxime apramycin, gentamicin and tetracycline) showed almost perfect agreement $(K>0.900)$ between phenotype and genotype. Fifty-seven percent of total $E$. coli isolates $(\mathrm{n}=295)$ had reduced susceptibility to ampicillin, and $96 \%$ showed $(n=283)$ correlation with a resistance genotype; isolates harboured one or more $\beta$-lactamase 
Strong and moderate correlation between phenotype and genotype of Escherichia coli isolates from pooled caecal contents of healthy pigs by antimicrobial, United Kingdom, $2018(\mathrm{n}=515)$

\section{A. Ciprofloxacin}

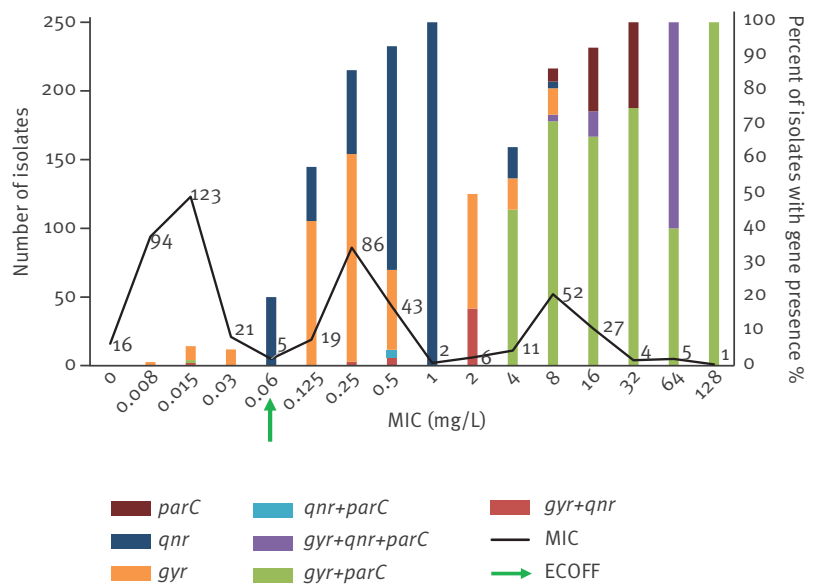

C. Florofenicol

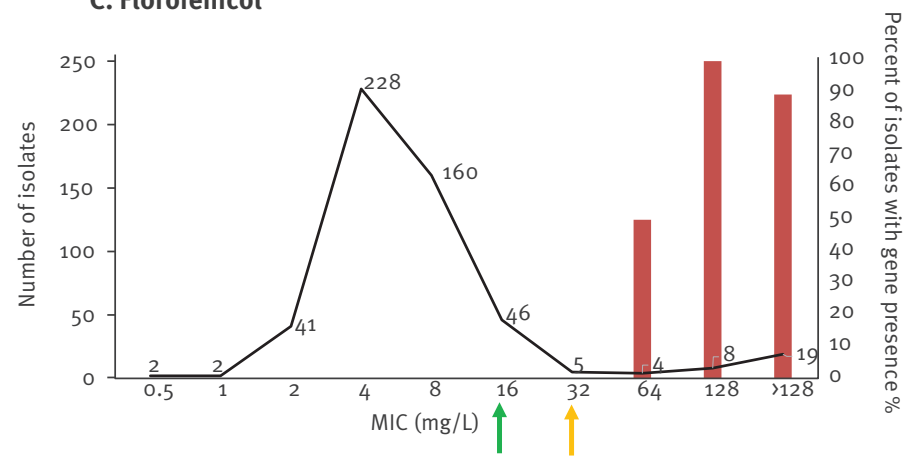

$\begin{aligned} \text { floR } & \rightarrow \text { ECOFF } \\ \text { MIC } & \rightarrow \text { Suggested cut-off }\end{aligned}$
B. Sulfamethoxazole:Trimethoprim (5:1)

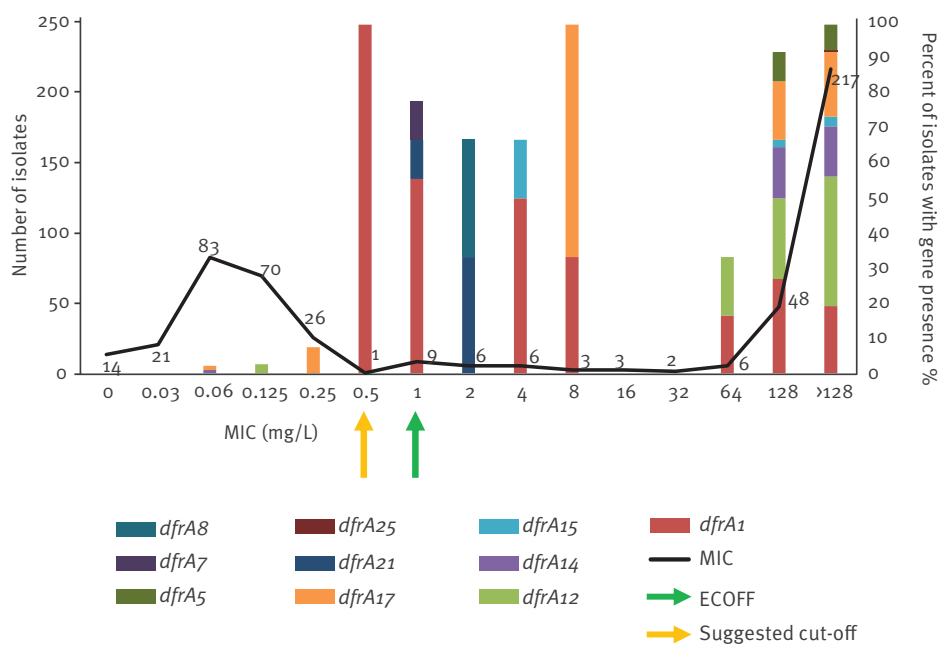

D. Ceftazidime

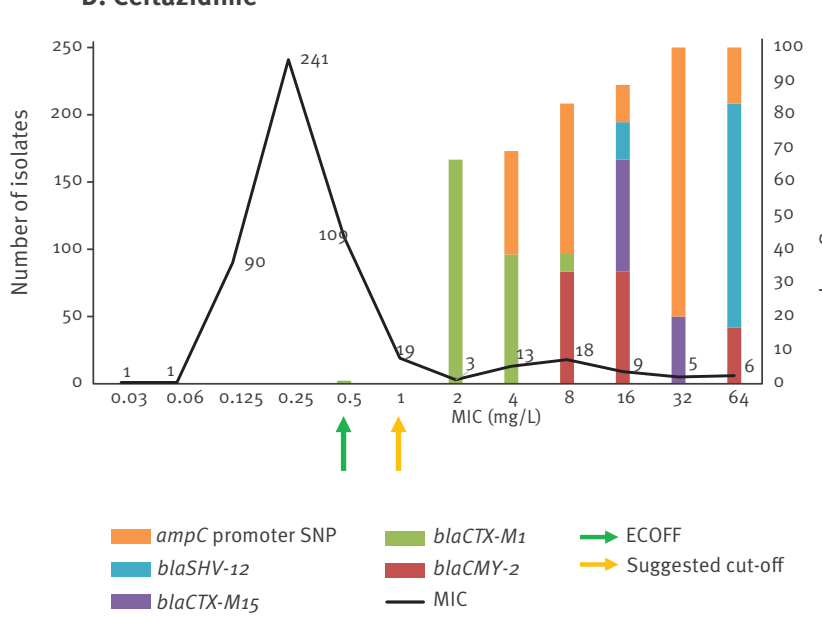

ECOFF: European Committee on Antimicrobial Susceptibility Testing epidemiological cut-off; MIC: minimum inhibitory concentration.

The MIC distribution and the underlying genetic mechanisms present in isolates are shown.

Florfenicol (C) is only used in veterinary medicine.

resistance genes and/or a chromosomal $\operatorname{ampC}$ promoter mutation (Table 1, Figure 1A). Two isolates were phenotypically susceptible to ampicillin but harboured $\beta$-lactamase resistance genes; one isolate contained a bla $a_{C T X-M-1}$ but was susceptible to ampicillin (MIC: 4 $\mathrm{mg} / \mathrm{L}$ ), ceftazidime (MIC: $0.5 \mathrm{mg} / \mathrm{L}$ ) and cefotaxime (MIC: $0.125 \mathrm{mg} / \mathrm{L}$ ), and the other ampicillin susceptible isolate (MIC: $2 \mathrm{mg} / \mathrm{L}$ ) possessed two bla $a_{T E M}$ variants (bla $a_{T E M-1 b}$ and bla $a_{T E M-30}$ ). For cefotaxime, 50 of 515 isolates (10\%) had reduced susceptibility by ECOFF, of which, 45 isolates harboured a genetic resistance determinant present in our APHA SeqFinder database (Figure 1B). Twenty-seven isolates harboured transferable extended spectrum $\beta$-lactamase (ESBL) resistance genes (Table
1) and 18 contained the chromosomal $\operatorname{ampC}$ promoter mutation, which can lead to mutational de-repression or constitutive expression of AmpC [25]. The remaining five isolates showing reduced susceptibility (MIC: $0.5^{-2} \mathrm{mg} / \mathrm{L}$ ), did not harbour an ESBL gene or chromosomal mutation in the $\operatorname{ampC}$ promoter.

For apramycin, using the DANMAP proposed breakpoint, there was $100 \%$ agreement between the phenotype and genotype; 23 isolates showed reduced susceptibility (MIC: $\geq 128 \mathrm{mg} / \mathrm{L}$ ) and harboured the aac3-Iva gene (Figure $1 \mathrm{C}$ ). Similarly for gentamicin there was almost perfect correlation $(\mathrm{K}=0.976)$, where 45 of 47 isolates with reduced susceptibility harboured 
a gentamicin resistance gene (Figure 1D). A further two isolates with reduced susceptibility (MIC: $\geq 16 \mathrm{mg} / \mathrm{L}$ ) did not harbour a gentamicin resistance gene; these isolates also showed reduced susceptibility to ampicillin (MIC: $\geq 128 \mathrm{mg} / \mathrm{L}$ ), florfenicol (MIC: $\geq 64 \mathrm{mg} / \mathrm{L}$ ) and sulfamethoxazole:trimethoprim (MIC: $>128 \mathrm{mg} / \mathrm{L}$ ), but did not harbour the associated resistance genes, suggesting a possible alternative multidrug resistance mechanism, such as efflux.

Reduced susceptibility to tetracycline was the most common in our $E$. coli collection ( $77 \%, \mathrm{n}=395$ isolates), and we were able to correlate this with a resistance genotype in 387 isolates which harboured a tet variant gene (Figure $1 \mathrm{E}$ ). Six phenotypically susceptible isolates also harboured a tet variant, four of these were at the ECOFF value (MIC: $8 \mathrm{mg} / \mathrm{L}$ ) and harboured either tet $(\mathrm{A})$ or tet $(\mathrm{C})$; two isolates with a MIC between 1 and $2 \mathrm{mg} / \mathrm{L}$ harboured tet(M), which is common in Gram-positive bacteria but has also been reported from E. coli and Salmonella [26,27].

\section{Strong agreement, kappa value 0.800 to 0.900} Three antimicrobials (ciprofloxacin, sulfamethoxazole:trimethoprim and florfenicol) showed very strong agreement $(\mathrm{K}=0.812$ to 0.848$)$ between the genotype and phenotype. Fifty percent $(n=256)$ of isolates showed reduced susceptibility to ciprofloxacin; 218 of these harboured a plasmid mediated quinolone resistance (PMQR) gene $(26 \%, n=57)$ and/or SNPs in the quinolone resistance determining regions (QRDR) of $g y r A$ or $\operatorname{parC}(74 \%, n=161)$. We noted that the MIC of isolates harbouring PMQR genes only $(n=57)$ was between 0.125 and $8 \mathrm{mg} / \mathrm{L}$, while those with QRDR mutations only $(n=152)$ ranged between 0.125 and $128 \mathrm{mg} / \mathrm{L}$. In the remaining 38 isolates with reduced susceptibility, no genotypic resistance mechanism was identified. Ten isolates were susceptible to ciprofloxacin but harboured genetic determinants; nine harboured mutations in the QRDR and one isolate harboured $q n r S_{1}$ and was at the ECOFF value (Figure 2A).

For sulfamethoxazole:trimethoprim, $57 \%$ isolates $(n=291)$ showed reduced susceptibility with 258 of these harbouring a $d f r$ variant alone $(n=90)$ or in combination with a sul $(n=168)$. Two isolates had a sul variant alone (Figure 2B). For the remaining 31 isolates with reduced susceptibility to sulfamethoxazole:trimethoprim, no significant match with genetic determinants in our database were detected. As presence solely of $d f r$ or in combination with sul resulted in reduced susceptibility; for phenotype and genotype correlation, these combinations were included and resulted in strong agreement $(\mathrm{K}=0.812)$. Fifteen susceptible isolates (5\%) harboured a $d f r$ gene alone $(n=10)$ or in combination with a sul $(n=5)$, seven of these were at the ECOFF ( 1 $\mathrm{mg} / \mathrm{L})$, and 37 susceptible isolates only harboured a sul variant.
Florfenicol is an antimicrobial solely used in veterinary medicine, and 36 isolates showed reduced susceptibility and resulted in strong agreement $(\mathrm{K}=0.848)$. The floR gene was present in 27 of these isolates with $M I C>32 \mathrm{mg} / \mathrm{L}$, but in the remaining nine, no florfenicol resistance determinants were detected (Figure $2 \mathrm{C}$ ). We noted that isolates harbouring $\mathrm{cml}$ or catA1 genes, which are associated with chloramphenicol resistance, did not confer cross-resistance to florfenicol, typically showing MIC value $32 \mathrm{mg} / \mathrm{L}$ (data not shown).

\section{Moderate agreement, kappa value 0.600 to 0.799}

The lowest agreement was observed with ceftazidime $(\mathrm{K}=0.726) ; 73$ isolates $(14 \%)$ showed reduced susceptibility using ECOFF, but only 45 isolates harboured an ESBL gene or chromosomal $a m p C$ promoter mutation. The remaining 28 isolates did not harbour a resistance gene or chromosomal changes present in our database (Figure 2D).

\section{Effect of adjusting the epidemiological cut-off} For florfenicol, others $[28,29]$ have suggested a ECOFF value for $E$. coli isolates tested from swine and cattle of $>32 \mathrm{mg} / \mathrm{L}$ as an alternative to the current one at>16 $\mathrm{mg} / \mathrm{L}$. When the higher MIC cut-off value was applied to our dataset only 31 of 515 (6\%) isolates showed reduced phenotypic susceptibility, and reduced the number of false negative isolates to four. The higher cut-off value also improved sensitivity and raised kappa correlation for florfenicol to the almost perfect correlation category, from 0.848 to 0.927 . A similar scenario was observed for tetracycline where lowering cut-off value from $>8 \mathrm{mg} / \mathrm{L}$ to $>4 \mathrm{mg} / \mathrm{L}$, (Figure $1 \mathrm{E}$ ) increased the kappa correlation from 0.930 to 0.945 so only two isolates were false positive. For eight isolates, the correlation did not change; they remained as false negative.

For sulfamethoxazole:trimethoprim, the MICs were determined using a 5:1 ratio that is relevant for veterinary medicine [30], however, the ECOFF value is based on a 19:1 ratio as used in human clinical settings. Based on the ratio of $5: 1$ sulfamethoxazole:trimethoprim used, we suggest that a lower ECOFF value of $>0.5$ $\mathrm{mg} / \mathrm{L}$ is more appropriate for the methodology applied here and will need to be tested for wider application (Figure 2B). However, its application increased the number of isolates with reduced susceptibility from 291 to 300 (58\%), with 210 of these isolates containing a $d f r$ variant. As a result, the number of susceptible isolates harbouring a $d f r$ gene halved, improving the kappa agreement between MIC phenotype and WGS gene presence from 0.812 to 0.836 . For ceftazidime, comparison of the MIC with WGS at a slightly higher cut-off of $>1 \mathrm{mg} / \mathrm{L}$ (Figure 2D) improved the kappa correlation, from 0.726 to 0.889 , because of a decrease in the number of false negative isolates.

Using the ECOFF cut-off values, the number of isolates that showed complete phenotype/genotype 
concordance for all nine antimicrobials in their susceptibility profile was 394 (77\%). For the remaining 121 isolates, there were discrepancies (false positive or false negative results) in up to five antimicrobials. Using the adjusted cut-off values for the four antimicrobials above, the numbers of isolates with genotype/phenotype concordance for all nine antimicrobials across their susceptibility profile increased to 419 (81\%), with the number of discrepant isolates decreasing to 96 .

\section{Discussion}

In this study, we explored the use of WGS data as an alternative to the traditional phenotypic method, MIC, used to determine AMR. At the time of this study, the BSAC agar dilution MIC was the accepted method for determining susceptibility, and in 2016, BSAC migrated to the EUCAST broth microdilution method in order to harmonise resistance prediction interpretations across Europe [19]. For this reason, EUCAST ECOFF values were applied to our data. A future study comparing the genotype results with phenotypes derived from broth microdilution would be of interest. However, as there was almost perfect agreement between WGS and MIC for the majority of antimicrobials when ECOFF values were used with agar dilutions, the improvements may or may not be substantial when using broth microdilutions. Additionally, there were no considerable changes to the kappa values obtained in this study when isolate WGS data were tested with other AMR pipelines available (data not shown).

Sulfamethoxazole:trimethoprim, florfenicol, ceftazidime and ciprofloxacin, which had the poorest agreement, nevertheless showed strong to moderate kappa values. However, when the ECOFF value was adjusted for several aforementioned antimicrobials, following analysis of the MIC distribution with gene presence, we noted an improvement in the kappa correlation, i.e. an increase in the number of isolates that were true positives and true negatives. The majority of discrepancies in our results were because of false negative correlations, i.e. isolates with reduced susceptibility lacking a relevant genetic resistance determinant from our database, suggesting that unknown resistance genes may be present in these bacteria. However, the overall WGS/MIC specificity of $99 \%$, the predictive value of a positive test being $98 \%$ and a kappa value of 0.930 was encouraging, and adds to the growing number of studies recommending the use of AMR genotyping $[10,12-14]$.

In other studies where $E$. coli WGS was compared with phenotype $[10,12,13]$, reported discrepancies mainly included phenotypically-susceptible isolates harbouring a resistance gene. In this study, a small number of such false positive isolates (14/31) had an MIC at or below the ECOFF value; this was most commonly observed for sulfamethoxazole:trimethoprim and ciprofloxacin. In the case of sulfamethoxazole:trimethoprim, determining the phenotype from genotype was complicated by the different mechanisms of resistance, including the expression of efflux pumps and cell wall permeability [31].

This study focused on known acquired resistance determinants. However, there are a number of other chromosomal genes where SNPs and variations in expression could result in resistance, e.g. folA-encoding dihydrofolate reductase for trimethoprim [11], or expression of multidrug efflux systems such as the mar operon $[32,33]$, that were not investigated and could explain some of the false negative results that were obtained. Also, there may be improvement in phenotypic and genotypic correlations if an ECOFF was attained appropriate for the 5:1 ratio used in veterinary medicine. Other limitations of the WGS approach are that only known genes can be determined $[1,6]$ and that it does not take genes that may be present but not expressed or the effect of multiple resistance genes present for the same class of antimicrobial into account [34].

However, WGS can be used to identify the presence of novel-acquired mechanisms to explain discrepancies, for example the identification of the pleuromutilin resistance gene tva(A) in Brachyspira spp. [35]. New resistance mechanisms can be added to the AMR database and the WGS can be screened again to perform retrospective analysis once new/novel genes are detected, as shown following $\mathrm{mcr}$ detection in 2015 [36]. Therefore, isolates found to have moderate kappa correlations in this study may be harbouring novel genes/mechanisms that require further investigation.

The EUCAST report on the role of WGS in AMR susceptibility testing of bacteria recommended that the primary MIC comparator for WGS predictions be the ECOFF, but it encouraged using the clinical breakpoints as a secondary comparator, acknowledging that doing so would be more challenging with our current knowledge [6]. Although our study found almost perfect correlation ( $\mathrm{K}>0.9$ ) between phenotype/genotype for most of the antimicrobials considered using ECOFF cut-offs, clinical breakpoints available for four antimicrobials showed much lower correlation (Supplementary Table S1). Clinical breakpoints are based on the success of treatment and therefore factors other than the MIC distribution alone are taken into consideration, e.g. host, clinical treatment data and pharmacokinetics of the drug [37]; properties that cannot be predicated from bacterial genome sequence alone.

WGS also provides the potential to predict susceptibility to a wider range of antimicrobials, including those not routinely tested in MIC panels. The APHA SeqFinder pipeline screens for 2,044 genes. In addition to the genes associated with the nine antimicrobials tested in this study (Table 1), a further 29 genes with predicted reduced susceptibility to other aminoglycosides (aac6-Ild, aad(various), ant3-1a, aph3, aph4, strA and $s t r B$ genes), chloramphenicol ( $\mathrm{cml}$, cat), streptothricin (sat2) and macrolides (ermB, inuF, mefB and $m p h A$ and $m p h B$ ) were also detected in the $515 E$. 
coli isolates. WGS data can also be used to provide further information on strains, including the multilocus sequence types, phylogeny, plasmids and plasmid types [36] that are important in assessing transmission of AMR. However, standardisation of WGS methodologies and analysis of the data is required before this technology is applied in AMR reference laboratories for routine surveillance activities.

In conclusion, the results of this study demonstrate that the use of WGS, a technological advancement over traditional phenotyping, should be considered an alternative way to monitor antimicrobial resistance in bacteria by national and pan-European surveillance programmes.

\section{Acknowledgements}

We thank Mr Jack Garrety for microbiology, Drs Miranda Kirchner and Luke Randall for guidance on MICs, and $\mathrm{Mr}$ Christopher Teale for his critical comments on the manuscript.

Funding statement: This work was funded by the Veterinary Medicines Directorate (VMD) to MFA under VMD grant number VMo533.

\section{Conflict of interest}

None declared.

\section{Authors' contributions}

Laboratory work: ES, MAb, ESa

Data analysis: ES, MAb, HOC, RC, MFA

Manuscript writing: ES, MAb, RC, MFA

Concept and funding: MFA

\section{References}

1. Anjum MF. Screening methods for the detection of antimicrobial resistance genes present in bacterial isolates and the microbiota. Future Microbiol. 2015;10(3):317-20. https://doi.org/10.2217/fmb.15.2 PMID: 25812454

2. Partridge SR, Kwong SM, Firth N, Jensen SO. Mobile Genetic Elements Associated with Antimicrobial Resistance. Clin Microbiol Rev. 2018;31(4):eo0088-17. https://doi.org/10.1128/ CMR.00088-17 PMID: 30068738

3. Punina NV, Makridakis NM, Remnev MA, Topunov AF. Wholegenome sequencing targets drug-resistant bacterial infections. Hum Genomics. 2015;9(1):19. https://doi.org/10.1186/s40246015-0037-Z PMID: 26243131

4. European Commission (EC). Commission Implementing Decision 2013/652/EU of 12 November 2013 on the monitoring and reporting of antimicrobial resistance in zonotic and commensal bacteria. L 303/26. 14 Nov 2013. Available from: https:// op.europa.eu/en/publication-detail/-/publication/83e1934f4d39-11e3-ae03-01aa75ed71a1/language-enhttps:// publications.europa.eu

5. European Food Safety Authority (EFSA)/European Centre for Disease Prevention and Control (ECDC).The European Union summary report on antimicrobial resistance in zoonotic and indicator bacteria from humans, animals and food in 2015. EFSA J. 2017;15(2):4694.

6. Ellington MJ, Ekelund O, Aarestrup FM, Canton R, Doumith $\mathrm{M}$, Giske $\mathrm{C}$, et al. The role of whole genome sequencing in antimicrobial susceptibility testing of bacteria: report from the EUCAST Subcommittee. Clin Microbiol Infect. 2017;23(1):2-22. https://doi.org/10.1016/j.cmi.2016.11.012 PMID: 27890457

7. Duggett NA, Sayers E, AbuOun M, Ellis RJ, Nunez-Garcia J, Randall $\mathrm{L}$, et al. Occurrence and characterization of mcr-1harbouring Escherichia coli isolated from pigs in Great Britain from 2013 to 2015. J Antimicrob Chemother. 2017;72(3):691-5. https://doi.org/10.1093/jac/dkw477 PMID: 27999032

8. McArthur AG, Waglechner N, Nizam F, Yan A, Azad MA, Baylay AJ, et al. The comprehensive antibiotic resistance database. Antimicrob Agents Chemother. 2013;57(7):3348-57. https:// doi.org/10.1128/AAC.00419-13 PMID: 23650175

9. Zankari E, Hasman H, Cosentino S, Vestergaard M, Rasmussen $\mathrm{S}$, Lund $\mathrm{O}$, et al. Identification of acquired antimicrobial resistance genes. J Antimicrob Chemother. 2012;67(11):2640-4. https://doi.org/10.1093/jac/dks261 PMID: 22782487

10. Do Nascimento V, Day MR, Doumith M, Hopkins KL, Woodford N, Godbole G, et al. Comparison of phenotypic and WGSderived antimicrobial resistance profiles of enteroaggregative Escherichia coli isolated from cases of diarrhoeal disease in England, 2015-16. J Antimicrob Chemother. 2017;72(12):328897. https://doi.org/10.1093/jac/dkx301 PMID: 28961934

11. Moran RA, Anantham S, Holt KE, Hall RM. Prediction of antibiotic resistance from antibiotic resistance genes detected in antibiotic-resistant commensal Escherichia coli using PCR or WGS. J Antimicrob Chemother. 2017;72(3):700-4. https://doi. org/10.1093/jac/dkw511 PMID: 28039273

12. Stoesser N, Batty EM, Eyre DW, Morgan M, Wyllie DH, Del Ojo Elias C, et al. Predicting antimicrobial susceptibilities for Escherichia coli and Klebsiella pneumoniae isolates using whole genomic sequence data. I Antimicrob Chemother. 2013;68(10):2234-44. https://doi.org/10.1093/jac/dkt18o PMID: 23722448

13. Tyson GH, McDermott PF, Li C, Chen Y, Tadesse DA, Mukherjee $S$, et al. WGS accurately predicts antimicrobial resistance in Escherichia coli. J Antimicrob Chemother. 2015;70(10):2763-9. https://doi.org/10.1093/jac/dkv186 PMID: 26142410

14. Zankari E, Hasman H, Kaas RS, Seyfarth AM, Agersø Y, Lund O, et al. Genotyping using whole-genome sequencing is a realistic alternative to surveillance based on phenotypic antimicrobial susceptibility testing. J Antimicrob Chemother. 2013;68(4):7717. https://doi.org/10.1093/jac/dks496 PMID: 23233485

15. AbuOun M, Stubberfield EJ, Duggett NA, Kirchner M, Dormer L, Nunez-Garcia J, et al. mcr-1 and mcr-2 variant genes identified in Moraxella species isolated from pigs in Great Britain from 2014 to 2015. J Antimicrob Chemother. 2017;72(10):2745-9. https://doi.org/10.1093/jac/dkx286 PMID: 29091227

16. Andrews JM. Determination of minimum inhibitory concentrations. J Antimicrob Chemother. 2001;48(Suppl 1):516. https://doi.org/10.1093/jac/48.suppl_1.5 PMID: 11420333

17. World Health Organization (WHO). Critically Important Antimicrobials for Human Medicine, 5 th revision. Geneva: WHO; 2017. Available from: https://www.who.int/foodsafety/ publications/antimicrobials-fifth/en/

18. European Committee on Antimicrobial Susceptibility Testing (EUCAST). Antimicrobial wild type distribution of microorganisms. Basel; EUCAST. [Accessed 2018]. Available from: htts://mic.eucast.org/Eucast2/.

19. Brown DF, Wootton M, Howe RA. Antimicrobial susceptibility testing breakpoints and methods from BSAC to EUCAST. J Antimicrob Chemother. 2016;71(1):3-5. https://doi. org/10.1093/jac/dkv287 PMID: 26377864

20. Danish Integrated Antimicrobial Resistance Monitoring and Research Programme (DANMAP). DANMAP 2004 - Use of antimicrobial agents and occurrence of antimicrobial resistance in bacteria from food animals, foods and humans in Denmark. Søborg: DANMAP; July 2005. Available from: https://www.danmap.org/-/media/arkiv/projekt-sites/danmap/ danmap-reports/danmap_2004.pdf?la=en

21. Bankevich A, Nurk S, Antipov D, Gurevich AA, Dvorkin M, Kulikov AS, et al. SPAdes: a new genome assembly algorithm and its applications to single-cell sequencing. J Comput Biol. 2012;19(5):455-77. https://doi.org/10.1089/cmb.2012.0021 PMID: 22506599

22. Card R, Zhang J, Das P, Cook C, Woodford N, Anjum MF. Evaluation of an expanded microarray for detecting antibiotic resistance genes in a broad range of gram-negative bacterial pathogens. Antimicrob Agents Chemother. 2013;57(1):458-65. https://doi.org/10.1128/AAC.01223-12 PMID: 23129055

23. Mackinnon A. A spreadsheet for the calculation of comprehensive statistics for the assessment of diagnostic tests and inter-rater agreement. Comput Biol Med. 2000;30(3):127-34. https://doi.org/10.1016/So0104825(00)00006-8 PMID: 10758228 
24. McHugh ML. Interrater reliability: the kappa statistic. Biochem Med (Zagreb). 2012;22(3):276-82. https://doi.org/10.11613/ BM.2012.031 PMID: 23092060

25. Lin CF, Hsu SK, Chen CH, Huang JR, Lo HH. Genotypic detection and molecular epidemiology of extended-spectrum beta-lactamase-producing Escherichia coli and Klebsiella pneumoniae in a regional hospital in central Taiwan. J Med Microbiol. 2010;59(Pt 6):665-71. https://doi.org/10.1099/ jmm.0.015818-0 PMID: 20150317

26. Figueiredo R, Card RM, Nunez-Garcia J, Mendonça N, da Silva GJ, Anjum MF. Multidrug-Resistant Salmonella enterica Isolated from Food Animal and Foodstuff May Also Be Less Susceptible to Heavy Metals. Foodborne Pathog Dis. 2019;16(3):166-72. https://doi.org/10.1089/fpd.2017.2418 PMID: 30480469

27. Bryan A, Shapir N, Sadowsky MJ. Frequency and distribution of tetracycline resistance genes in genetically diverse, nonselected, and nonclinical Escherichia coli strains isolated from diverse human and animal sources. Appl Environ Microbiol. 2004;70(4):2503-7. https://doi.org/10.1128/ AEM.70.4.2503-2507.2004 PMID: 15066850

28. Rayamajhi N, Cha SB, Kang ML, Lee SI, Lee HS, Yoo HS. Interand intraspecies plasmid-mediated transfer of florfenicol resistance in Enterobacteriaceae isolates from swine. Appl Environ Microbiol. 2009;75(17):5700-3. https://doi. org/10.1128/AEM.02816-08 PMID: 19592530

29. Singer RS, Patterson SK, Meier AE, Gibson JK, Lee HL, Maddox CW. Relationship between phenotypic and genotypic florfenicol resistance in Escherichia coli. Antimicrob Agents Chemother. 2004;48(10):4047-9. https://doi.org/10.1128/ AAC.48.10.4047-4049.2004 PMID: 15388477

30. European Agency for the Evaluation of Medicinal Products (EMEA). Committee For Veterinary Medicinal Products. Trimethoprim. Summary Report (2). London; EMEA: 1997 Available from: https://www.ema.europa.eu/en/documents/ mrl-report/trimethoprim-summary-report-2-committeeveterinary-medicinal-products_en.pdf

31. Huovinen P. Resistance to trimethoprim-sulfamethoxazole. Clin Infect Dis. 2001;32(11):1608-14. https://doi. org/10.1086/320532 PMID: 11340533

32. Maneewannakul K, Levy SB. Identification for mar mutants among quinolone-resistant clinical isolates of Escherichia coli. Antimicrob Agents Chemother. 1996;40(7):1695-8. https://doi. org/10.1128/AAC.40.7.1695 PMID: 8807064

33. Speer BS, Shoemaker NB, Salyers AA. Bacterial resistance to tetracycline: mechanisms, transfer, and clinical significance. Clin Microbiol Rev. 1992;5(4):387-99. https://doi.org/10.1128/ CMR.5.4.387 PMID: 1423217

34. Hopkins KL, Batchelor MJ, Anjum M, Davies RH, Threlfall EJ. Comparison of antimicrobial resistance genes in nontyphoidal salmonellae of serotypes enteritidis, hadar, and virchow from humans and food-producing animals in England and wales. Microb Drug Resist. 2007;13(4):281-8. https://doi. org/10.1089/mdr.2007.779 PMID: 18184054

35. Card RM, Stubberfield E, Rogers J, Nunez-Garcia J, Ellis RJ, AbuOun M, et al. Identification of a New Antimicrobial Resistance Gene Provides Fresh Insights Into Pleuromutilin Resistance in Brachyspira hyodysenteriae, Aetiological Agent of Swine Dysentery. Front Microbiol. 2018;9:1183. https://doi. org/10.3389/fmicb.2018.01183 PMID: 29971045

36. Duggett NA, Randall LP, Horton RA, Lemma F, Kirchner M, Nunez-Garcia J, et al. Molecular epidemiology of isolates with multiple mcr plasmids from a pig farm in Great Britain: the effects of colistin withdrawal in the short and long term. J Antimicrob Chemother. 2018;73(11):3025-33. https://doi. org/10.1093/jac/dky292 PMID: 30124905

37. Turnidge J, Paterson DL. Setting and revising antibacterial susceptibility breakpoints. Clin Microbiol Rev. 2007;20(3):391408. https://doi.org/10.1128/CMR.00047-06 PMID: 17630331

\section{License, supplementary material and copyright}

This is an open-access article distributed under the terms of the Creative Commons Attribution (CC BY 4.0) Licence. You may share and adapt the material, but must give appropriate credit to the source, provide a link to the licence and indicate if changes were made.

Any supplementary material referenced in the article can be found in the online version.

This article is copyright of the authors or their affiliated institutions, 2019. 